\title{
Alcohol-free EUS-guided chemoablation of multiple pancreatic insulinomas
}

\section{다)(1) $\odot$}

Authors

Daniel Gaballa ${ }^{1}$, Catherine S. Abendroth ${ }^{2}$, Matthew T. Moyer ${ }^{1}$

Institutions

1 Department of Internal Medicine, Division of Gastroenterology, Penn State University Hospital, Hershey Medical Center, Hershey, Pennsylvania, United States

2 Department of Pathology, Division of Anatomic Pathology, Penn State University Hospital, Hershey Medical Center, Hershey, Pennsylvania, United States

submitted 13.2.2018

accepted after revision 25.6.2018

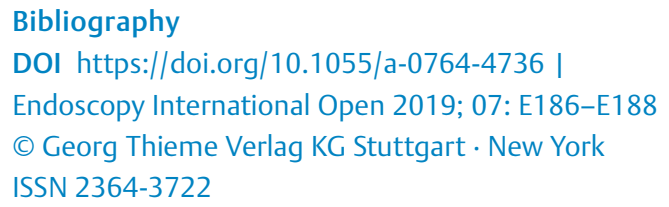

Corresponding author

Daniel Gaballa, MD, Department of Internal Medicine, Penn State University Hospital, Hershey Medical Center, Hershey, PA 17033

Fax: +1-717-531-5831

dgaballa@pennstatehealth.psu.edu

\section{ABSTRACT}

Background and study aims Endoscopic ultrasound (EUS)-guided chemoablation with ethanol lavage, followed by infusion with paclitaxel, has been found to be effective for treatment of mucinous pancreatic cysts. However, there are notable adverse events (AEs) associated with ethanol and its undesirable inflammatory effects on local tissue and vessels. The recent ChARM trial demonstrated that removing ethanol from the cyst ablation process resulted in equivalent efficacy while significantly reducing associated AEs. Encouraged by these results, we speculated that alcohol-free chemoablation can be applied to treatment of solid tumors, as described in our case with a patient with severe and symptomatic recurrent hypoglycemia in the setting of multifocal insulinomas. As a result, the patient saw a significant reduction in symptoms. EUS-guided alcohol-free chemoablation may represent a new alternative to previously established therapies that will ultimately reduce risk of AEs.

\section{Introduction}

Neuroendocrine tumors (NET) affect the gastrointestinal system with a frequency of 3.65 per 100,000 population [1] but arise ubiquitously throughout the body. Insulinomas, one of the more common types of gastrointestinal NET, are typically found in or adjacent to the pancreas and are characterized by inappropriate endogenous secretion of insulin. These hormonally active NETs often cause refractory hypoglycemia that leads to significant morbidity [2,3], but they typically have low potential for metastasis [3].

Unfortunately, for many patients with NETs, medical therapies result in variable efficacy and can result in significant side effects. If these lesions are localized, surgical resection is effective or even curative. However, such treatment has morbidity and mortality rates of $20 \%$ to $40 \%$ and $1 \%$ to $5 \%$, respectively, and its own limitations and surgery often is not an option in elderly patients or those with significant comorbidities. This has led to increasing interest in minimally invasive treatment options of NETs, and of these, EUS-guided ablation with alcohol may be a promising technique $[4,5]$.

Previous reports of alcohol-based ablation have shown good efficacy but with a serious adverse events (SAEs) rate of $6 \%$ [4], including post-procedure pancreatic injury with pseudocysts, acute pancreatitis, hematomas, and ulceration of the duodenal wall $[6,7]$. SAEs associated with EUS-guided ablation generally have been felt to be secondary to the marked inflammatory and toxic effects of dehydrated alcohol, which can extravasate into local tissues $[5,8,9]$. This has called into question its clinical application and safety profile. A recent randomized prospective clinical trial conducted by this group demonstrated that alcohol is not required for EUS-guided pancreatic cyst ablation when a chemotherapy infusion cocktail specifically designed for pancreatic neoplasia is used, and that when alcohol is removed 
from the ablation process, AE rates fall significantly [9]. By extrapolation of these data, we speculated that solid tumor ablation would not only be comparatively effective, but also safer, if the same admixture used in the CHARM trial was used in place of alcohol. Here we describe use of an alcohol-free chemoablation of multifocal insulinomas in a patient who was not a surgical candidate.

\section{Case report}

In 2004, a 49-year-old-female presented with recurrent symptomatic hypoglycemia. Triphasic enhanced computed tomography (CT) of the abdomen and pelvis showed two lesions consistent with insulinomas in the distal tail of the pancreas. The woman underwent a distal pancreatectomy and splenectomy with surgical pathology revealing a multifocal well-differentiated neuroendocrine tumor, Grade 1 ( $\mathbf{F i g} \mathbf{1}$ ). There were a total of four subcentimeter tumor nodules. Immunohistochemical staining demonstrated insulin production by the neoplastic cells. The patient was subsequently discharged with close follow up with Endocrinology for further medical management with diazoxide and octreotide.

Unfortunately, over the last 3 years, the patient had a recurrence of hypoglycemic episodes that were not responsive to medical therapy, and for which she was forced to self-treat by frequently sipping soft drinks, resulting in 29-kg weight gain and depression. She was hospitalized with refractory hypoglycemia in June of 2016 with CT imaging showing only postsurgical changes to the pancreas without any visible masses to suggest recurrent insulinoma. Endoscopic ultrasound-guided fineneedle aspiration (EUS-FNA) was performed in July 2016 with findings of three subcentimeter lobular masses $(9 \mathrm{~mm} \times 9 \mathrm{~mm})$ in the head and body of the pancreas. Color Doppler imaging was utilized to guide FNA of the largest mass lesion with cytology diagnostic for recurrent neuroendocrine tumor ( The patient was discharged with increased titration of her octreotide in addition to a cornstarch regimen. She could not continue the diazoxide due to side effects of excessive fluid retention. Despite these medication changes, the woman continued to have recurrent hypoglycemic episodes.

Because of the multifocal nature of the patient's lesions and a body mass index (BMI) of $57.81 \mathrm{~kg} / \mathrm{m}^{2}$, state of immobility, and comorbidities such as chronic kidney disease, the Surgical Oncology team felt that a surgical approach posed an excessive mortality risk. The patient was referred to Gastroenterology for consideration of EUS-guided chemo-ablation using the alcoholfree chemoablation admixture previously described by this group for ablation of mucinous-type pancreatic cysts. The patient was fully informed of all her options as well as the risks and benefits of the procedure.

The procedure was performed in an operating room due to a high-risk airway and a BMI of $57.81 \mathrm{~kg} / \mathrm{m}^{2}$. Under general anesthesia, a linear EUS video endoscope (GF-UCT180; Olympus America, Center Valley, Pennsylvania, United States) was used to perform a full pancreaticobiliary examination. The three known NETs ( $\mathbf{F i g . 3}$ ), as well as a fourth lesion that had developed in the interim period, were treated by injecting 1 to 2 cc of

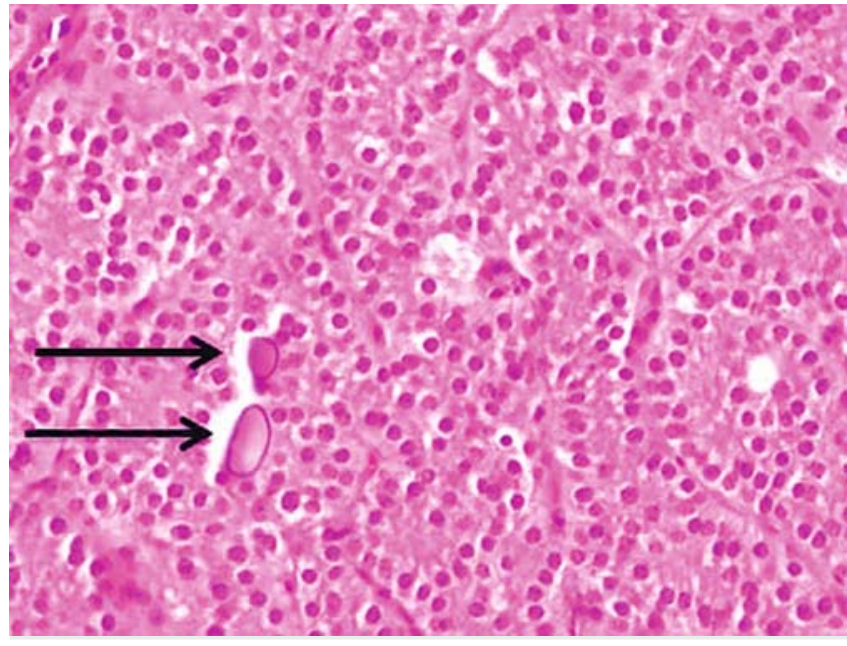

- Fig. 1 Resection specimen of well-differentiated neuroendocrine tumor, Grade 1, characterized by cords and trabeculae of mitotically-inactive cells with small, uniform nuclei having typical "salt and pepper" chromatin and inconspicuous nucleoli. There were frequent psammoma body type calcifications (arrow) (H\&E × 400).

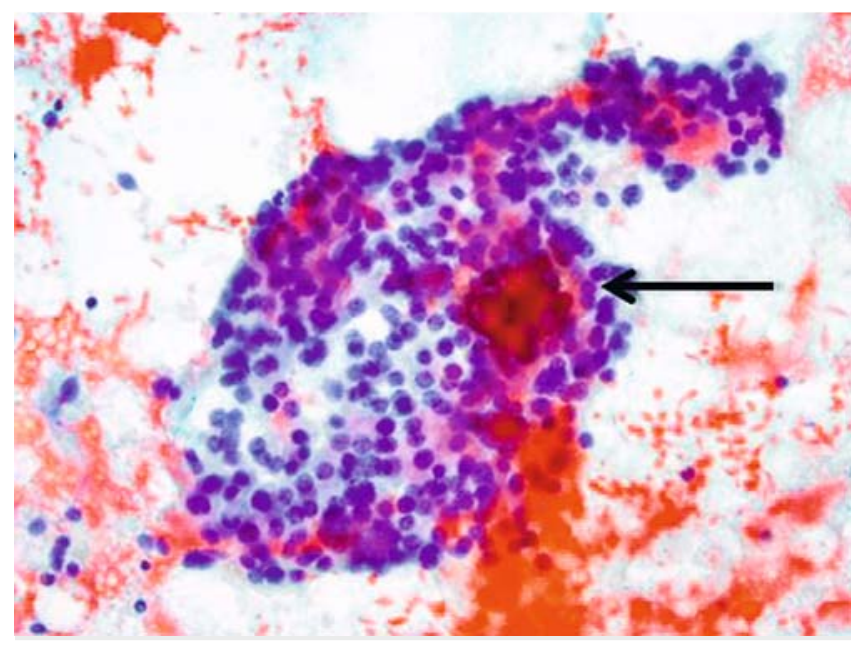

- Fig. 2 Direct smear from EUS-FNA showing cells with identical cytomorphology, with small, uniform nuclei, "salt and pepper" chromatin, and inconspicuous nucleoli, and with associated psammoma body type calcifications (arrow) (Papanicolaou stain, × 400).

the 3-mg paclitaxel and $19 \mathrm{mg}$ of gemcitabine admixture into the center of each mass using a 22-g FNA needle (Echotip; Cook Medical, Bloomington, Indiana, United States). Repeat EUS 3 months later identified only two masses consistent with insulinomas and two associated pathological lymph nodes, which were all treated, utilizing a total of $5 \mathrm{~mL}$ of the chemoablation cocktail. The patient maintained appropriate hemodynamics and experienced acceptable post-procedure pain with no major complications during either procedure.

At follow-up 3 months later, the patient said she felt subjectively markedly improved. Since her last ablation, reported that she has not required any emergency care for her symptoms. She previously reported that she would have several episodes 


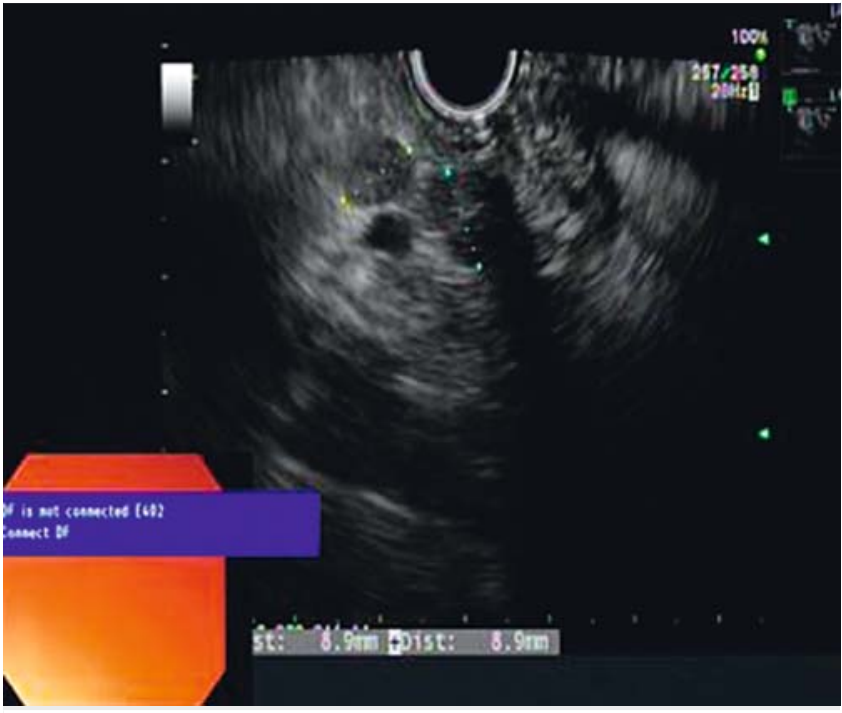

- Fig. 3 EUS with color Doppler imaging noting three lobular masses lobular masses in the head and body of the pancreas.

of severe, symptomatic hypoglycemia daily, which has now been reduced to only three to five episodes monthly. Several of the medications to control her symptoms have since been reduced or discontinued all together.

\section{Conclusion}

EUS-guided alcohol ablation of hormonally active NETs is effective; however, rates of SAEs of $6 \%$, felt to be secondary to the inflammatory and toxic effects of alcohol, are a limitation. Recent trials have shown that with mucinous pancreatic cyst ablation, alcohol is not required when an admixture of gemcitabinepaclitaxel is used in its place, and that when alcohol is removed from the ablation process, SAE rates fall significantly [5, 9]. In this limited case report, we have shown that alcohol-free ablation of NETs can also be safe and effective. Although promising, it is unclear if alcohol-free EUS-guided chemoablation of solid NETs represents an advance in treating these lesions in patients who are not good surgical candidates. However, further investigation is certainly warranted given the possible equivalent efficacy and improved safety when compared to alcohol-based ablation.

\section{Competing interests}

Dr. Moyer is a consultant for Boston Scientific.

\section{References}

[1] Lawrence B, Gustafsson BI, Chan A et al. The epidemiology of gastroenteropancreatic neuroendocrine tumors. Endocrinol Metab Clin North Am 2011; 40:

[2] Hirshberg B, Cochran C, Skarulis M et al. Malignant insulinoma. Cancer 2005; 104: $264-272$

[3] Mathur A. The Surgical clinics of North America: Insulinoma. WB Saunders Company; 2009; 89: 1105

[4] Moyer MT, Sharzehi S, Abraham M et al. The safety and efficacy of an alcohol-free pancreatic cyst ablation protocol. Gastroenterology 2017; 153: 1295-1303

[5] Moyer MT, Dye CE, Sharzehi S et al. Is alcohol required for effective pancreatic cyst ablation? The prospective randomized CHARM trial pilot study Endosc Intl Open 2016; 4: E603 - E607

[6] Levy M], Thompson GB, Topazian MD et al. US-guided ethanol ablation of insulinomas: a new treatment option. Gastrointest Endosc 2012; 75: $200-206$

[7] Deprez PH, Claessens A, Borbath I et al. Successful endoscopic ultrasound-guided ethanol ablation of a sporadic insulinoma. Acta Gastroenterol Belg 2008; 71: 333-337

[8] DeWitt J, McGreevy K, Schmidt CM et al. EUS-guided ethanol versus saline solution lavage for pancreatic cysts: a randomized, doubleblind study. Gastrointest Endosc 2009; 70: 710-723

[9] Oh HC, Seo DW, Lee TY et al. New treatment for cystic tumors of the pancreas: EUS-guided ethanol lavage with paclitaxel injection. Gastrointest Endosc 2008; 67: 636-642 